$\overline{A E E T}$

ASOCIACIÓN ESPAÑOLA DE ECOLOGÍA TERRESTRE

\title{
Modelos ecológicos: descripción, explicación y predicción
}

\author{
J.A. Blanco ${ }^{1, *}$ \\ (1) Departamento de Ciencias del Medio Natural, Universidad Pública de Navarra, 31006, Pamplona, Navarra, España. \\ * Autor de correpondencia: J.A. Blanco [juan.blanco@unavarra.es]
}

> Recibido el 16 de diciembre de 2013, aceptado el 18 de diciembre de 2013.

Blanco, J.A. 2013. Modelos ecológicos: descripción, explicación y predicción. Ecosistemas 22(3):1-5. Doi.: 10.7818/ECOS.2013.22-3.01.

\section{La ecología: una ciencia descriptiva, explicativa y predictiva}

Los ecosistemas son, por definición, complejos sistemas en los que factores biológicos, climáticos y geológicos se combinan con factores sociales, políticos y económicos para producir una serie de procesos ambientales que determinan la evolución de cada uno de estos ecosistemas en el tiempo. Esto hace que el estudio y la gestión de cualquier ecosistema sea un asunto siempre complejo. Por ello, cualquier herramienta que permita simplificar el estudio de estos factores y predecir los efectos de alterar alguno de ellos es de gran ayuda tanto en la investigación como en la gestión de los ecosistemas (Blanco 2013).

La aplicación de la ciencia de la ecología a la investigación y gestión de los ecosistemas requiere del abordaje de tres componentes complementarios: conocer, entender y predecir. "Conocer" es análogo a una descripción de un fotograma. "Entender" es análogo a examinar los componentes de cada fotograma y cómo encajan unos con otros. "Predecir" es hacer una película reuniendo los fotogramas que proporcionan fotos estáticas del sistema en diferentes momentos. Cada una de estas partes es necesaria para la resolución de problemas complejos, y ninguna es suficiente de forma aislada. Solamente cuando se combinan las tres partes se pueden alcanzar los objetivos y proporcionar una base adecuada para guiar la compleja relación entre la humanidad y los recursos naturales (Fig. 1). La comunicación es considerada una cuarta parte de la ciencia, ya que la ciencia que no se comunica no contribuye al avance de la humanidad en la búsqueda de una forma de vida más sostenible con nuestro ambiente. La comunicación es requerida en los tres componentes de la ciencia, y este monográfico de Ecosistemas es parte de ese esfuerzo por parte de los científicos para comunicar sus hallazgos.

\section{Conocer}

El primer paso para resolver un problema es conocerlo, comparándolo con otros problemas, objetos o condiciones. Este conocimiento se genera por medio del proceso de inducción, que básicamente consiste en pasar de descripciones de muchos ejemplos del problema, objeto o condición a conclusiones que se deriven de los mismos. Este tipo de conocimiento basado en la experiencia es más fiable como base para la gestión que un simple sistema de creencias que carezca de esa experiencia. Sin embargo, el conocimiento y la experiencia siempre se relacionan con el pasado y el presente, respectivamente, mientras que la gestión de los ecosistemas requiere conocer qué pasará en el futuro. El poder del componente inductivo de la ciencia es que si el conocimiento se basa en muchos ejemplos u observaciones de la realidad que provienen de situaciones complejas, esa complejidad subyacente está incorporada de forma implícita. Sin embargo, la experiencia por sí sola puede ser una base adecuada (y a veces la mejor) para predecir el futuro siempre que el éste sea idéntico, o muy similar, al pasado. Sin embargo, este no es generalmente el caso. De hecho, muchos problemas en la gestión de recursos naturales aparecen cuando el público desea que se cambien las políticas y formas de gestión por otras nuevas de las que se carece de experiencia. Dos tipos de actividad científica se requieren para proporcionar suficiente confianza en nuestras interpretaciones basadas en la experiencia antes de que puedan usarse como unos cimientos fiables para una gestión efectiva. El primero es una prueba crítica de esa experiencia y de las hipótesis que genera. Este proceso proporciona entendimiento o comprensión del ecosistema (Kimmins et al. 2010). El segundo es una extrapolación de ese entendimiento a otras situaciones, siguiendo un proceso que genera predicciones sobre posibles estados alternativos de los ecosistemas.

\section{Entender}

Entender un problema, objeto o condición supone reducir la complejidad de las explicaciones o teorías iniciales basadas en la inducción a unidades más pequeñas. Este es el proceso de reduccionismo en que se basa la ciencia. Después se utiliza un proceso de deducción para generar hipótesis sobre esos componentes que puedan ser probadas experimentalmente. Una síntesis de esos resultados proporciona una base para una predicción fiable. En la ecología básica los resultados experimentales producidos a partir de investigaciones de componentes y procesos individuales se añaden al creciente conocimiento científico. Este conocimiento sirve a su vez de cimientos para nuevas teorías o postulados sobre esos procesos complejos (un procedimiento inductivo). Después de que esta rueda inductiva-deductiva (Fig. 1) dé varias vueltas se pueden generar principios científicos, y si estos principios sobreviven a largos periodos de pruebas pueden llegar a convertirse en leyes científicas. Sin embargo, esos principios y leyes suelen ser teóricos y reduccionistas (refiriéndose a los componentes más simples de un ecosistema), por lo que es difícil aplicarlos directamente como la base para la resolución de problemas complejos en la gestión o investigación de los ecosistemas naturales. Entender los componentes y procesos individuales de un problema, objeto o condición es un componente necesario de la ciencia, pero no es suficiente para resolver problemas complejos, hace falta algo más: predicciones. 


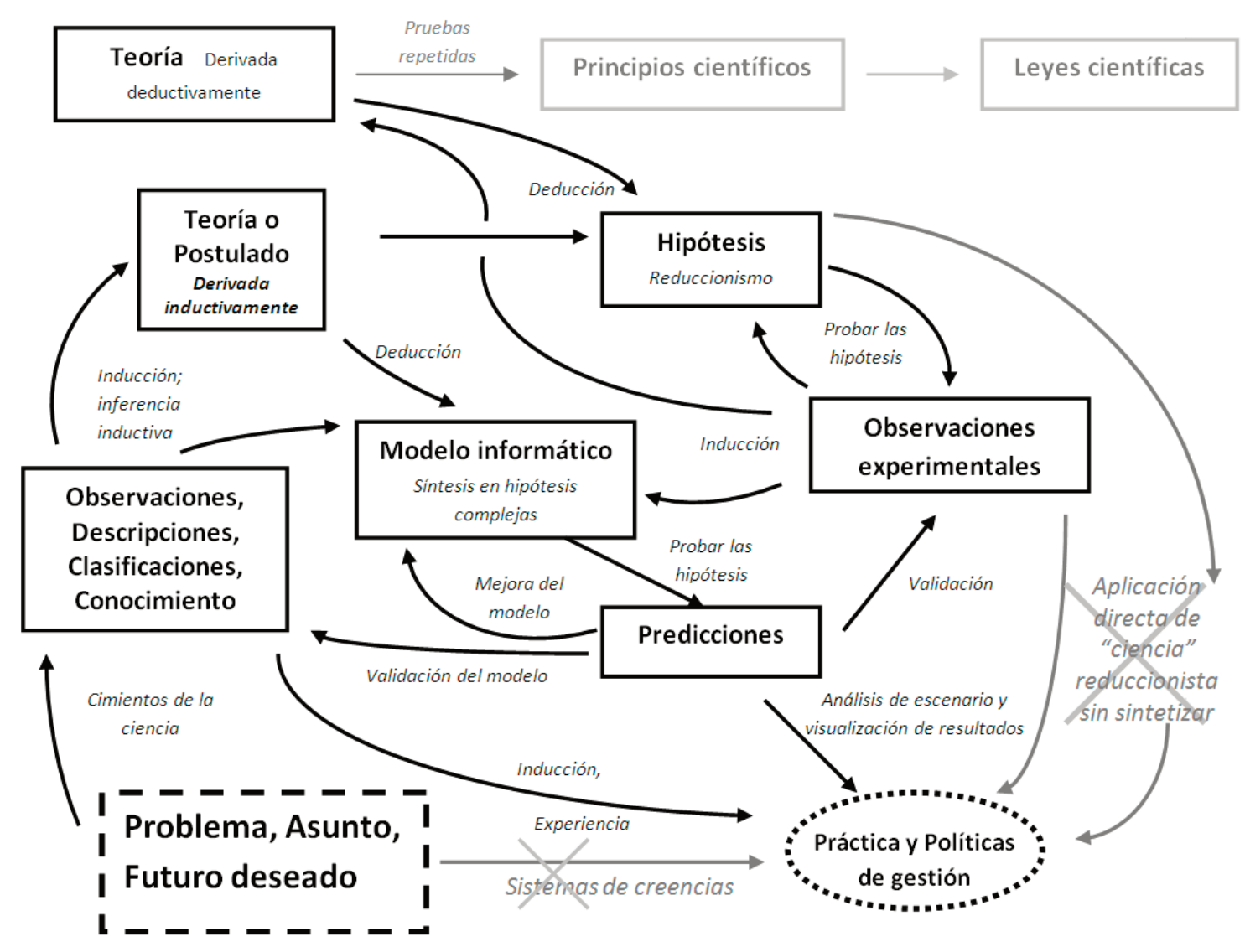

Figura 1. Los componentes principales de una ciencia completa en la gestión de recursos naturales. Nótese que ni un sistema de creencias poco informado ni la aplicación directa de los resultados de la ciencia hipotético-deductiva sin sintetizar (el componente de entendimiento) proporciona una base adecuada para la práctica y la política del manejo de recursos naturales (adaptado de Kimmins et al. 2005).

\section{Predecir}

Predecir es la capacidad de coordinar el conocimiento acumulado para explorar posibles situaciones en el futuro. En esencia, predecir es meramente el proceso de extrapolar el conocimiento y entendimiento del pasado y el presente en el futuro. Las predicciones están típicamente basadas en relaciones definidas entre variables predictivas y variables respuesta. Describir, entender y cuantificar esas relaciones es lo que ha empujado el bucle inductivo-deductivo de prueba de hipótesis (Fig.1). La aplicación de la ciencia en la resolución de problemas ambientales siempre necesita de alguna forma de predicción basada en una síntesis del conocimiento y entendimiento de los componentes del sistema (Kimmins et al. 2010). En su nivel más básico, la predicción en ciencia necesita la creación de un modelo mediante un proceso en el que se piensa sistemáticamente sobre un problema (Jakeman et al. 2008). Por tanto, desarrollar un modelo ecológico supone la organización de datos, asunciones y conocimiento para un propósito específico. Utilizada como una forma de entender sistemas complejos, la modelización ecológica puede tener muchas formas (Kimmins 2004).

\section{Modelos en ecología}

Los modelos son simplificaciones de la realidad que están diseñadas para analizar algunos aspectos del sistema real que tratan de emular. La creación de un modelo está intrínsecamente unida con el problema en el cual el modelizador está interesado. En este monográfico de Ecosistemas se presentan varios tipos de modelos ecológicos, desde "simples" modelos empíricos hasta complejos modelos de simulación socio-ecológica. Los modelos se utilizan cuando es más simple trabajar con un substituto que con el sistema real completo (Ford 1999). Son útiles porque ayudan al usuario de estos modelos a aprender algo sobre el sistema que representan, y a tomar decisiones relacionadas con el sistema real sin tener que tratar con toda su complejidad. Sin embargo, cuanto más simple es el modelo comparado con el sistema real, menos información contiene y por lo tanto menos útil es para desarrollar nuevas ideas de investigación y gestión ecológicas. Por otro lado, cuanto más simple es un modelo, más rápidamente se pueden probar teorías o políticas de gestión que pueden ser o no adecuadas, y que pueden ser estudiadas con más detalle después. Sin embargo, cuando esta simplificación omite información sobre la respuesta de los ecosistemas a distintos factores ambientales o de manejo, los resultados de los modelos son con frecuencia engañosos.

Los ecólogos siempre deben estar al tanto de los peligros de la sobre-simplificación. En otras palabras, hay que recordar que aunque en ciencia se utiliza la "navaja de Occam" (o el principio de parsimonia) para escoger la explicación (o modelo) más simple capaz de explicar el fenómeno de estudio, la navaja tiene dos filos, ya que como decía Albert Einstein, "un modelo tiene que ser tan simple como sea posible, pero tan complejo como sea necesario". La aportación de Polo (2013), en este número, es una magnífica discusión de cómo un modelo matemático muy simple debe ir volviéndose paulatinamente más complejo para explicar las pautas observadas en poblaciones reales de animales en las cuales conviven individuos con distintos tipos de estrategias para obtener los mismos recursos necesarios para su supervivencia.

El grado de complejidad de un modelo depende por lo tanto del objetivo para el cual el modelo ha sido desarrollado (Kimmins et al. 2008). Hay muchos objetivos específicos que pueden abordarse con la construcción de un modelo ecológico. Los modelos pueden utilizarse para: 1) representar variables y tasas de cambio; 2) describir la estructura de un ecosistema y los patrones temporales y espaciales de procesos ecosistémicos individuales; 3 ) reconstruir el pasado o predecir el comportamiento futuro del ecosistema estudiado; 4) generar y probar teorías e hipótesis ecológicas sobre la organización y funcionamiento de los ecosistemas; 5) mostrar, codificar, transferir, evaluar e interpretar el conocimiento ecológico; 6) guiar el desarrollo y evaluación de políticas ambientales; 7) facilitar el aprendizaje colectivo y resolver disputas (Morton 1990, Beven 
2002); y 8) educar y enseñar conceptos ecológicos. Como puede apreciarse, la mayoría puede agruparse en una de las dos categorías principales: explicar cómo funciona o está constituido un ecosistema o predecir algunas variables concretas cuando se producen unos determinados valores de factores ambientales.

Los modelos que están diseñados para la predicción sin necesidad de explicaciones sobre cómo funciona el ecosistema pueden ser tan simples como una ecuación que resuma un conjunto de variables y que responda a la pregunta de "¿Cuánto cambia $X$ cuando Y varía?”. Un ejemplo de este tipo de modelos ecológicos lo presentan en este número González-Izquierdo et al. (2013), al desarrollar un modelo que explica el potencial de crecimiento del pino caribeño (Pinus caribaea Morelet) en el oriente cubano en función de las características del suelo. Este modelo es una herramienta útil para el desarrollo de un manejo forestal sostenible en Cuba, pero las relaciones entre las variables sólo se mantienen en la localización para la cual ha sido desarrollado el modelo. Por lo tanto, aunque la metodología es transferible a otros lugares, los detalles de este tipo de modelos no lo son (Mangel et al. 2001). Este tipo de modelos empíricos puede necesitar de análisis complejos, entre los que destacan las posibilidades de utilizar análisis multivalentes para explorar las interacciones entre factores ambientales, descritas en este número por Ledo et al. (2013).

Por otro lado, cuando el objetivo del ecólogo es explicar cómo funciona un ecosistema, es necesaria una descripción específica de los procesos ecológicos y como están unidos entre sí. Aunque tales modelos explicativos pueden ser generales y aplicables a un amplio rango de ecosistemas (p.ej. un libro de texto de ecología que explique las interacciones entre la estructura y la función de los ecosistemas), si lo que los modelizadores pretenden es aportar información para apoyar una gestión sostenible o una investigación detallada, será necesario proporcionar valores numéricos para explorar relaciones cuantitativas entre distintas variables. Esta es una fase importante del proceso de modelización: clarificar lo que es conocido y qué necesita más estudio.

Como en otras ramas de las ciencias ambientales, los modelos no se suelen usar para un único objetivo. Por lo tanto, la distinción entre modelos predictivos y explicativos con frecuencia está poco clara. En ecología, muchos modelos se crean resumiendo grandes bases de datos de observaciones de campo en parcelas permanentes, utilizando relaciones empíricas entre factores ambientales. Ejemplos de este tipo de modelos se revisan en el trabajo de Ruiz-Benito et al. (2013), en el cual se discuten opciones de modelización para estimar la vulnerabilidad de los bosques ibéricos al cambio climático. Un ejemplo concreto de la aplicación de uno de estos modelos lo proporcionan Peralta e Imbert (2013), quienes muestran la potencialidad de la distribución de la carrasca (Quercus Ilex L.) en Navarra. También es notable la contribución de Gómez-Aparicio et al. (2013), quienes revisan la utilidad en ecología de los modelos de vecindad.

Los modelos que se desarrollan con la intención de predecir los posibles estados futuros del ecosistema en cuestión deben estar basados en la representación de los procesos ecológicos clave que son relevantes para el problema de interés para el modelizador. En general, estos modelos que simulan mecanismos (y por lo tanto también llamados mecanicistas) proporcionan un conocimiento más profundo del funcionamiento de los ecosistemas que los modelos empíricos. Un buen ejemplo de este tipo de modelos es GOTILWA+, un modelo desarrollado para estudiar la influencia de la disponibilidad de agua y otros recursos en el crecimiento de ecosistemas forestales, descrito por Nadal-Sala et al. (2013) en este número. Sin embargo, este mayor entendimiento no se refleja necesariamente en unas predicciones más ajustadas a la realidad, ya que los modelos mecanicistas por lo general son más difíciles de calibrar e incorporan más incertidumbre que los modelos empíricos. Este problema lo exploran con detalle Gárate y Blanco (2013), quienes investigan la sensibilidad del modelo de ecosistemas forestales FORECAST a la incertidumbre asociada a la calibración de los parámetros relacionados con la biomasa de raíces en bosques de pino silvestre (Pinus sylvestris L.) de los Pirineos.
La principal característica de los modelos mecanicistas (o también llamados de procesos) es que pueden utilizarse como modelos exploratorios para predecir como un ecosistema (o una parte del mismo) podría funcionar si existe un cambio en las condiciones ambientales (ecológicas o socio-económicas). Esto permite la utilización de los modelos ecológicos como un banco de pruebas donde realizar experimentos "virtuales" que pueden guiar tanto la investigación futura como los planes de gestión de los ecosistemas estudiados. Buenos ejemplos de este tipo de modelos los proporcionan las contribuciones en este número de Baños-González et al. (2013) y Martínez-Fernández et al. (2013), quienes estudian la dinámica de sistemas socio-ecológicos en la reserva de la biosfera de Fuerteventura (Islas Canarias) y en las zonas agrícolas del Mar Menor (Murcia, España oriental), respectivamente.

En la práctica, todos los modelos tienen algún grado de empiricismo: utilizan datos de campo para resumir procesos ecofisiológicos a escalas espaciales o temporales más pequeñas que a las escalas que funciona el modelo en cuestión. Reconociendo esta realidad, el desarrollo de un tipo de modelos intermedio entre mecanicistas y empíricos (los llamados modelos híbridos; Kimmins 2004, Blanco 2012) está ganando importancia en el campo de la gestión ecosistémica. Estos modelos incorporan elementos causales y empíricos al mismo nivel jerárquico (Johnsen et al. 2001). El desarrollo de estos modelos se está popularizando porque se ha mostrado que los modelos empíricos pueden mejorarse al incorporar algunas funciones teóricas o causales clave (Monserud 2003), y que los modelos mecanicistas pueden mejorar al incorporar limitantes y elementos empíricos a nivel de ecosistema (Mäkelä et al. 2000).

La selección de cualquiera de las opciones de modelización anteriores depende del objetivo específico seleccionado por el modelizador (ver la contribución en este número de Ruiz-Benito et al. 2013). Se podría pensar que en ciencia se favorecerán los modelos más mecanicistas debido a su poder explicativo y su capacidad para explorar las consecuencias de condiciones ambientales futuras cambiantes (Giske et al. 1992, Giske 1998). Sin embargo, si el interés está en predicciones a corto plazo, los usuarios o diseñadores de modelos ecológicos pueden preferir los modelos más empíricos.

Por último, los modelos ecológicos no deben considerarse como trabajos aislados, ya que siendo una de sus principales características el ayudarnos a entender cómo funcionan los ecosistemas, un modelo "finalizado" suele considerarse como un modelo "muerto", ya que no incorpora los cambios que a través de su uso se descubra que puedan ser adecuados (Kimmins 2004). En este sentido, también es posible la unión de distintos modelos que funcionen a distintas escalas, con el objetivo de aprovechar el trabajo realizado por otros investigadores y seguir avanzando en la compresión de los procesos ecológicos (Bonet-García et al. 2013). De esta forma, en los últimos años están apareciendo más esfuerzos de "meta-modelos", en los que varios modelos están unidos de forma secuencial, de forma que los resultados de un modelos son las entradas del siguiente. Otra opción son los "mega-modelos" en los que varios modelos funcionan conectados de forma simultánea (Blanco 2012).

\section{Buenas prácticas en modelización ecológica}

Ya hay una literatura bien desarrollada sobre cómo crear modelos para la ecología y el manejo de recursos sostenibles. Los lectores interesados en el tema pueden consultar Box (1979), Starfield y Bleloch (1991), Vanclay (1994), Hilborn y Mangel (1997), Ford (1999), Shenk y Franklin (2001), Kimmins et al. (2010) o Fath and Jorgensen (2011), entre otros. No es la intención de esta editorial repetir esta información, pero no se debe dejar pasar la ocasión de mencionar la necesidad de observar un código de buenas prácticas a la hora de crear modelos. Las ideas mencionadas aquí pueden completarse con las guías proporcionadas por Van Waveren et al. (1999) y el Consejo para la Regulación de la Modelización Ambiental de los EE.UU. (CREM 2009), pero es oportuno incidir en ellas. 
Con la disponibilidad de grandes bases de datos cubriendo largos períodos de tiempo y con el mayor poder de computación de los ordenadores y programas actuales, el número de modelos ecológicos ha aumentado dramáticamente (Monserud 2003, Kimmins et al. 2010). El resultado ha sido una plétora de modelos, formas de describirlos y probarlos, y métodos para evaluar sus predicciones. Debido a esta situación, la necesidad de estandarizar los procedimientos de modelización ambiental está siendo cada día mayor (Van Weveren et al. 1999, CREM 2009). Además, un uso consistente y común de una terminología es necesario para la comunicación de avances en el desarrollo de modelos y en su evaluación. Terminología nueva o confusa debería ser claramente definida, y cualquier redefinición de terminología ya en uso debería tener un propósito claro (Crout et al. 2008). Como mucha de la teoría de modelización tiene sus orígenes en literatura estadística, los modelizadores deberían en lo posible utilizar la terminología original (ver el glosario en Ripley 1996). Terminología adicional relacionada con la modelización ambiental puede encontrarse en CREM (2009). Un componente clave de unas buenas prácticas de modelización es la documentación del modelo, y la puesta a disposición de esta documentación para que otros usuarios y expertos puedan revisarla. Bonet-García et al. (2013) muestran en este número una iniciativa muy interesante de creación de un repositorio de modelos, que esperamos sirva como referencia a otros esfuerzos futuros que se realicen en esta línea.

El primer aspecto clave es especialmente importante: contar con una buena documentación de los modelos ecológicos cuando el usuario del modelo es distinto a la persona que lo desarrolló. Los fallos de los modelos ecológicos pueden ser causados por un modelo conceptual incompleto o malentendido, una formulación incorrecta del modelo, o errores en la codificación del modelo. Por otro lado, este tipo de problemas debe distinguirse de errores a la hora de utilizar el modelo, que pueden causarse por un completo (o inexistente) manual de usuario, descuido en el tratamiento de los datos de entrada, calibración y evaluación insuficientes, o el uso del modelo más allá del alcance para el que fue diseñado. Estos problemas conducen a un bajo grado de fiabilidad de las predicciones del modelo (Van Waveren et al. 1999). Crout et al. (2008) aseveran que el principal requisito de un modelo es que se especifique claramente su propósito. ¿Para qué es el modelo? ¿Cuál es la razón para crear un modelo nuevo? ¿Qué se espera que haga el modelo? En ausencia de una definición del propósito del modelo, el grado de éxito del mismo no se puede juzgar, y tampoco se puede definir el nivel de complejidad estructural que es necesaria.

El segundo aspecto clave en una buena práctica de modelización es describir claramente los datos de campo y cómo se usan esos datos en el modelo, todas las asunciones y formulaciones del modelo. Un listado explícito de las asunciones revela el proceso de pensamiento del modelizador y facilita la evaluación de esas asunciones más tarde (Kimmins et al. 2010). Además, el modelizador debería proporcionar una justificación breve pero explícita de sus asunciones, y documentar las referencias bibliográficas que las describen en detalle, incluyendo cualquier preferencia subjetiva y opiniones (Wagener et al. 2003, Bonet-García et al. 2013).

Un tercer elemento clave en una buena práctica de modelización es la evaluación del modelo, la cual debería ser un proceso continuo. Además, se deben reconocer las diferencias entre evaluar las asunciones del modelo, implementar el modelo y evaluar el rendimiento del mismo. Este proceso incluye estudios de la sensibilidad del modelo a distintos parámetros (ver un ejemplo de esta práctica en Gárate y Blanco (2013), en este número), así como la comparación de las estimaciones del modelo con observaciones independientes de la misma variable. La evaluación del modelo, que tradicionalmente implica alguna medida de rendimiento predictivo y la incertidumbre asociada, debería ser una parte central del proceso de desarrollo del modelo, y no algo considerado únicamente tras la finalización del modelo. Aunque existen muchos métodos utilizados para evaluar modelos (ver por ejemplo Lo et al. 2011), aún no existe un proceso estándar para llevar a cabo este proceso. En cualquier caso, la fase de evaluación debería incluir una evaluación de los datos utilizados para compararlos con las predicciones del modelo, y una discusión de si las pautas y patrones de procesos ecológicos tienen sentido desde un punto de vista ecológico, y no solamente numérico (Blanco et al. 2007).

Para terminar, en este número de Ecosistemas también se informa sobre los últimos acontecimientos en España relacionados con los modelos ecológicos. A destacar es la reciente organización del $7^{\circ}$ Curso Internacional sobre Modelización Ecológica en Ciudad Real por la Universidad de Castilla-La Mancha, que ha contado con la presencia del Dr. S.E. Jørgensen, una autoridad mundial en modelos ecológicos y que ha sido apoyado por la Asociación Española de Ecología Terrestre (AEET) (ver http://www.aeet.org/7th_International_course_on_ECOLOGICAL_MODELLING_266_p.htm). También acaba de publicarse el libro "Aplicaciones de modelos ecológicos a la gestión de recursos naturales", editado por J.A. Blanco y publicado por Omnia Science (ver reseña en este número por Quero (2013)).

\section{Referencias}

Banos-González, I., Martínez-Fernández, J., Esteve, M.A. 2013. Simulación dinámica de sistemas socio-ecológicos: sostenibilidad en Reservas de la Biosfera. Ecosistemas 22(3): 74-83.

Beven, K. 2002. Towards a coherent philosophy for modelling the environment, Proceedings Royal Society of Series A 458:1-20.

Blanco J.A. (ed.). 2013. Aplicaciones de modelos ecológicos en la gestión de recursos naturales. Omnia Science, Barcelona. España. 210 pp. ISBN 978-84-940624 9-0.

Blanco, J.A. 2012. Más allá de los modelos de crecimiento:modelos ecológicos híbridos en el contexto del manejo forestal sostenible. Cuadernos de la Sociedad Española de Ciencias Forestales 34: 11-25

Blanco, J.A., Seely, B., Welham, C., Kimmins, J.P., Seebacher, T.M. 2007. Testing the performance of FORECAST, a forest ecosystem model, against 29 years of field data in a Pseudotsuga menziesii plantation. Canadian Journal of Research 37:1808-1820.

Bonet-García, F.J., Pérez-Pérez, R., Benito, B.M., Pérez-Luque, A.J., Zamora, R.J. 2013. Documentación de modelos y flujos de trabajo: el siguiente reto en el manejo de información ecológica. Ecosistemas 22(3): 37-45

Box, G.E.P. 1979. Robustness in scientific model building. In: R. L. Launer and G. N.Wilkinson (eds.) Robustness in statistics. Academic Press. New York, USA.

Council for Regulatory Environmental Modeling (CREM) 2009. Guidance on the Development, Evaluation, and Application of Environmental Models, Publication EPA/100/K-09/003, U.S. Environmental Protection Agency, Washington, D.C, USA.

Crout, N., Kokkonen, T., Jakeman, A.I., Norton, J.P., Newham, L.T.H., Anderson, R., Assaf, H., Croke, B.F.W., Gaber, N., Gibbons, J., Holzworth, D., Mysiak, J., Reichl, J., Seppelt, R., Wagener, T., Whitfield, P. 2008. Good modelling practice, In: A.J. Jakeman, A.J. Voinov, A.A., Rizzoli, E., Chen, S.H. (eds.). Environmental modelling, software and decision support: state of the art and new perspectives. Elsevier, Amsterdam, The Netherlands.

Fath B, Jørgensen S.E. 2011. Fundamentals of ecological modelling 4th Edition. Applications in Environmental management and Research. Elsevier, Dordetch, The Netherlands.

Ford, A. 1999. Modeling the Environment, Island Press, Washington, DC. USA.

Gárate, M., Blanco, J.A. 2013. Importancia de la caracterización de la biomasa de raíces en la simulación de ecosistemas forestales. Ecosistemas 22(3): 66-73.

Giske, J. 1998. Evolutionary models for fisheries management. In: Pitcher T., Pauly D., Hart P.J.B. (eds.). Re-inventing fisheries management, Chapman and Hall, Nueva York, USA.

Giske, J., Skjoldal, H.R., Aksnes, D.L. 1992. A conceptual model of distribution of capelin in the Barents Sea, Sarsia 77:147-156.

Gómez-Aparicio, L., Avila, J.M., Cayuela, L. 2013. Métodos de máxima verosimilitud en ecología y su aplicación en modelos de vecindad. Ecosistemas 22(3): 12-20.

González-Izquierdo, E., Barrero-Medel, H., Carrasco-Rodríguez, Y. 2013. Evaluación de las clases de calidad de sitio de Pinus caribaea var. caribaea en la Empresa Forestal Integral Macurije (Pinar del Río, Cuba). Ecosistemas 22(3): 46-51. 
Hilborn, R., Mangel, M. 1997. The ecological detective: Confronting models with data, Princeton University Press, Princeton, NJ, USA.

Jakeman, A.J., Chen, S.H., Rizzoli, A.E., Voinov, A.A. 2008. Modelling and software as instruments for advancing sustainability. In: A.J. Jakeman, A.J. Voinov, A.A., Rizzoli, E., Chen, S.H. (eds.). Environmental modelling, software and decision support: state of the art and new perspectives, Elsevier, Amsterdam, The Netherlands.

Johnsen, K., Samuelson, L., Teskey, R., McNulty, S., Fox, T. 2001. Process models as tools in forestry and management, Forest Science 47: 2-8.

Kimmins, J.P. 2004. Ecology. A foundation for sustainable management and environmental ethics in forestry, Pearson/Prentice Hall. Upper Saddle River, NJ. USA.

Kimmins, J.P., Blanco, J. A., Seely, B., Welham, C., Scoullar, K. 2008. Complexity in modelling forest ecosystems. How much is enough?. Ecology and Management 256: 1646-1658.

Kimmins, J.P., Blanco, J.A., Seely, B., Welham, C., Scoullar, K. 2010. Forecasting Futures: A Hybrid Modelling Approach to the assessment of sustainability of forest ecosystems and their values. Earthscan Ltd. London, UK. 281 pp. ISBN: 978-1-84407-922-3.

Kimmins, J.P., Welham, C., Seely, B., Meitner, M., Rempel, R., Sullivan, T. 2005. Science in forestry: why does it sometimes disappoint or even fail us?, The Forestry Chronicle 81: 723-734.

Ledo, A., Montes, F., Cañellas, I. 2013. Nuevas herramientas para el estudio de la interacción entre especies en el espacio y en el tiempo. Ecosistemas 22(3): 52-57.

Lo, Y.-H., Blanco, J.A., Seely, B., Welham, C., Kimmins, J.P. 2011. Generating reliable meteorological data in mountainous areas with scarce presence of weather records: the performance of MTCLIM in interior British Columbia, Canada. Environmental Modelling and Software 26: 644-657.

Mäkela, A., Landsberg, J., Ek, A.R., Burk, T.E., Ter-Mikaelian, M., Ågren, G.I., Oliver, C.D., Puttonen, P. 2000. Process-based models for fores ecosystem management: current state of the art and challenges for practical implementation, Tree Physiology 20: 289-298.

Mangel, M., Fiksen, Ø, Gisk, J. 2001 Theoretical and statistical models in natural resource management and research, In: Shenk, T.M., Franklin A.B. (eds). Modeling in natural resource management: development, interpretation, and application, Island Press, Washington, DC. USA.
Martínez-Fernández, J., Fitz, C., Esteve Selma, M.A., Guaita, N., MartínezLópez, J. 2013. Modelización del efecto de los cambios de uso del suelo sobre los flujos de nutrientes en cuencas agrícolas costeras: el caso del Mar Menor (Sudeste de España). Ecosistemas 22(3): 84-94.

Monserud, R.A. 2003. Evaluating forest models in a sustainable forest management context, FBMIS 1, 35-47.

Morton, A. 1990. Mathematical modelling and contrastive explanation, Canadian Journal of Philosophy 16, 251-270.

Nadal-Sala, D., Sabaté, S., Gracia, C. 2013. GOTILWA+: un modelo de procesos que evalúa efectos del cambio climático en los bosques y explora alternativas de gestión para su mitigación. Ecosistemas 22(3): 29-36.

Peralta, J., Imbert, J.B. 2013. ¿Puede haber carrascales en el sur de Navarra? Una aproximación mediante la modelización de distribución de especies. Ecosistemas 22(3): 58-65.

Polo, V. 2013. Modelos matemáticos en ecología: aplicación al dilema halcón vs. paloma. Ecosistemas 22(3): 6-11.

Quero, J.L. 2013. "Aplicaciones de modelos ecológicos a la gestión de recursos naturales" de Juan A. Blanco. 2013. Ecosistemas 22(3): 136.

Ripley, B.D. 1996. Pattern recognition and neural networks, Cambridge University Press, Cambridge, UK.

Ruiz-Benito, P., Herrero, A., Zavala, M.A. 2013. Vulnerabilidad de los bosques españoles frente al Cambio Climático: evaluación mediante modelos. Ecosistemas 22(3): 21-28.

Shenk, T.M., Franklin A.B. (eds). 2001. Modeling in natural resource management: Development, interpretation, and application, Island Press, .

Starfield, A.M., Bleloch, A.L. 1991. Building models for conservation and wildlife management, 2nd edition, Interaction Book Company, Edina, MN. USA.

Vanclay, J.K. 1994. Modelling Forest Growth and Yield: Applications to Mixed, CAB International, Wallingford, UK.

Van Waveren, R.H., Groot, S., Scholten, H., Van Geer, F.C., Wösten, J.H.M., Koeze, R.D., Noort, J.J. 1999. Good modelling practice handbook, STOWA report 99-05, Utrecht, The Netherlands.

Wagener, T., Wheater, H.S., Gupta H.V. 2003. Identification and evaluation of watershed models, In: Duan, Q., Sorooshian, S., Gupta, H.V., Rousseau., A., Turcotte, R. (eds.). Calibration of watershed models, American Geophysical Union, Washington DC. USA. 